\title{
Distribution and Biogeochemical Properties of Hydrothermal Plumes in the Rodriguez Triple Junction
}

\author{
Takuroh Noguchi, Tatsuhiro Fukuba, Kei Okamura, Akira ljiri, Katsunori \\ Yanagawa, Yoshiyuki Ishitani, Teruo Fujii, and Michinari Sunamura
}

\begin{abstract}
In 2010, we conducted seven surveys for the deep-sea hydrothermal plume through conductivity-temperature-depth profiler (CTD) "tow-yo" cast in the area of the Kairei field. We observed a turbidity anomaly with a maximum thickness of $120 \mathrm{~m}$, the upper limit of which was at $2,150 \mathrm{~m}$ water depth, approximately $300 \mathrm{~m}$ above the Kairei field hydrothermal vents $(\sim 2,440 \mathrm{~m})$. The depth of upper limit of turbidity anomaly around Kairei field was the same height as in previous reports. Because the maximum height of hydrothermal plumes are regulated by the density (temperature and salinity) of the end-member hydrothermal fluid and dilution by the ambient seawater, the height of the plume suggested that the hydrothermal activity of the Kairei field was also the same as 17 years ago. Deep sequencing of microbial 16S rRNA genes showed that the SUP05 phylotypes and Epsilonproteobacteria, which are known as the potential sulfur oxidizer and/or possibly hydrogen oxidizer, were propagated in the early stage of the hydrothermal plume and in the hydrothermal fluid-seawater mixing zone near the Kairei hydrothermal vents. Our exploration found a hydrothermal plume at $14 \mathrm{~km}$ north of the Kairei field, which had different $\mathrm{H}_{2} / \mathrm{CH}_{4}$ ratio expected from the end-member hydrothermal fluid of Kairei field and the ambient seawater mixing. The north plume had a lower $\mathrm{H}_{2}$, higher $\mathrm{CH}_{4}$ concentration, and higher microbial cell density than those in the hydrothermal plume around Kairei field. The north hydrothermal plume represented too oxic condition to harbor methane production by anaerobic methanogens. In addition, our microbial community structure analysis based on deep sequencing of $16 \mathrm{~S}$ rRNA genes more than 10,000-amplicon reads per one sample showed no signal of methanogenic archaea. This suggests little in situ methanogenesis from $\mathrm{H}_{2}$ in the plume. It seems likely that high concentration of methane in the north plume is derived from another hydrothermal plume source rather than the Kairei hydrothermal fluids. Further studies will be needed to understand the cause of high methane concentration in the north plume.
\end{abstract}

The online version of this chapter (doi:10.1007/978-4-431-54865-2_15) contains supplementary material, which is available to authorized users.

T. Noguchi $(\bowtie)$

Center for Advanced Marine Core Research, Kochi University, B200 Monobe, Nankoku, Kochi 783-8502, Japan

Marine Technology and Engineering Center, Japan Agency for Marine-Earth Science and Technology, 2-15 Natsushima-cho, Yokosuka, Kanagawa 237-0061, Japan

e-mail: noguchitk@jamstec.go.jp

T. Fukuba

Marine Technology and Engineering Center, Japan Agency for

Marine-Earth Science and Technology, 2-15 Natsushima-cho,

Yokosuka, Kanagawa 237-0061, Japan
K. Okamura

Center for Advanced Marine Core Research, Kochi University, B200 Monobe, Nankoku, Kochi 783-8502, Japan

A. Ijiri • K. Yanagawa $\bullet$ M. Sunamura

Department of Earth and Planetary Science, The University of Tokyo, 7-3-1 Hongo, Bunkyo-ku, Tokyo 113-0033, Japan

Y. Ishitani

Institute of Biogeoscience, Japan Agency for Marine-Earth Science and Technology, 2-14 Natsushima-cho, Yokosuka, Kanagawa 237-0061, Japan

T. Fujii Institute of Industrial Science, The University of Tokyo, 4-6-1 Komaba, Meguro-ku, Tokyo 153-8505, Japan 
Keywords

Hydrothermal plume observation - Microbial population and communities - Plume evolution • Rapid $\mathrm{H}_{2}$ consumption

\subsection{Introduction}

Once the venting hydrothermal fluid is sufficiently diluted, it forms a hydrothermal plume with neutral buoyancy that is spread by deep-sea currents. Although the potential density of a neutrally buoyant plume is mostly the same as that of the ambient seawater, some chemical species (e.g., dissolved methane $\left(\mathrm{CH}_{4}\right)$, helium $(\mathrm{He})$, and manganese (Mn) and physical properties (e.g., water temperature and turbidity) remain much higher in the hydrothermal plume than in the ambient seawater. In situ sensors and onboard- and onshore-analyses of water samples have been applied to detect these anomalies based on the previous studies of hydrothermal plume surveys (e.g., Baker 1990). These previous studies demonstrated the spatial distribution of hydrothermal plume and the gradation of chemical species. During a spreading process of a hydrothermal plume, chemical composition and stable isotopic composition changes which is termed "chemical evolution of plume". The chemical evolution of a plume is implicated by absorption to particulate materials, sedimentation, and oxidation (German and Von Damm 2003). During the oxidation process in the oxygenated seawater, microbes play an important role for $\mathrm{Mn}^{2+}, \mathrm{CH}_{4}$, and $\mathrm{NH}_{4}{ }^{+}$(e.g. Lam et al. 2004). Raised microbial cell densities in the hydrothermal plumes, compared to in the surrounding ambient seawater, have been reported at several hydrothermal fields (e.g., northern East Pacific Rise, Winn et al. (1986); north Fiji basin, Naganuma et al. (1989)).

During the TAIGA project, we focused on chemical fluxes from hydrothermal vents and its influences to deepsea ecosystems through a hydrothermal plume investigation. The Kairei field is one of the important targets in our project because the hydrothermal fluids of the Kairei field are characterized by high concentration of $\mathrm{H}_{2}$. In this chapter, we show the temporal and spatial distribution of chemical species in the hydrothermal plume, and the interaction between the chemical species and the microbial communities base on seven "tow-yo" surveys with conductivity-temperature-depth profiler (CTD) and our developed in situ chemical sensors, together with the water sampling and analysis of geochemistry and microbiology.

\subsection{Site Description}

Two hydrothermal fields have been discovered on the Central Indian Ridge: the Kairei field $\left(25^{\circ} 19.17^{\prime} \mathrm{S}, 70^{\circ} 02.40^{\prime} \mathrm{E}\right.$; 2,450 m water depth) (Hashimoto et al. 2001; Gamo et al. $2001)$ and the Edmond field $\left(23^{\circ} 52.68^{\prime} \mathrm{S}, 69^{\circ} 35.80^{\prime} \mathrm{E}\right.$; 3,290-3,320 m water depth) (Van Dover et al. 2001) (Fig. 15.1a). The geological settings and geochemical characteristics of these hydrothermal fields are described in this book (Okino et al. Chap. 11, Nakamura and Takai, Chap. 12). The end-member chemical and isotopic composition of hydrothermal fluid from the Kairei and Edmond fields are shown in Suppl. 15.1 (supplementary data) summarized from Gallant and Von Damm (2006) and Kumagai et al. (2008). The $\mathrm{H}_{2}$ concentration of the hydrothermal fluid from the Kairei field is higher than that from the Edmond field or other silicic- to mafic-rock-hosted hydrothermal fields (e.g., East Pacific Rise). Kumagai et al. (2008) and Nakamura et al. (2009) proposed that the high $\mathrm{H}_{2}$ concentration was derived from the interaction between the hydrothermal fluid and olivine-rich ultramafic rocks.

\subsection{Methods}

\subsubsection{In Situ Sensor Observations and Sampling}

Various sensors and water samplers have been used so far in hydrothermal vent surveys (e.g., Baker 1990). During the KH10-6 cruise of R/V Hakuho-maru in November 2010, we conducted seven CTD tow-yo surveys in the area of the Kairei field (Fig. 15.1b). In each survey, the following in situ sensors were used: a conductivity-temperature-depth profiler with Carousel Multi-Sampling system (CTDTCMS; SBE 9plus CTD and SBE 32 Carousel Water Sampler, Sea-Bird Electronics Inc.), a backscatter turbidity meter (Seapoint Sensors, Inc.), a glass electrode $\mathrm{pH}$ sensor ( $\mathrm{pH}-$ 08, Kimoto Electric Co., Ltd.), an ion-selective fieldeffective transistor pH sensor (Shitashima et al. 2002), oxidation-reduction potential (ORP) sensors (ORP-08 and ORP-09, Kimoto Electric Co., Ltd.), and a manganese analyzer (TANSAKUN Mn-08, Kimoto Electric Co., Ltd.) 


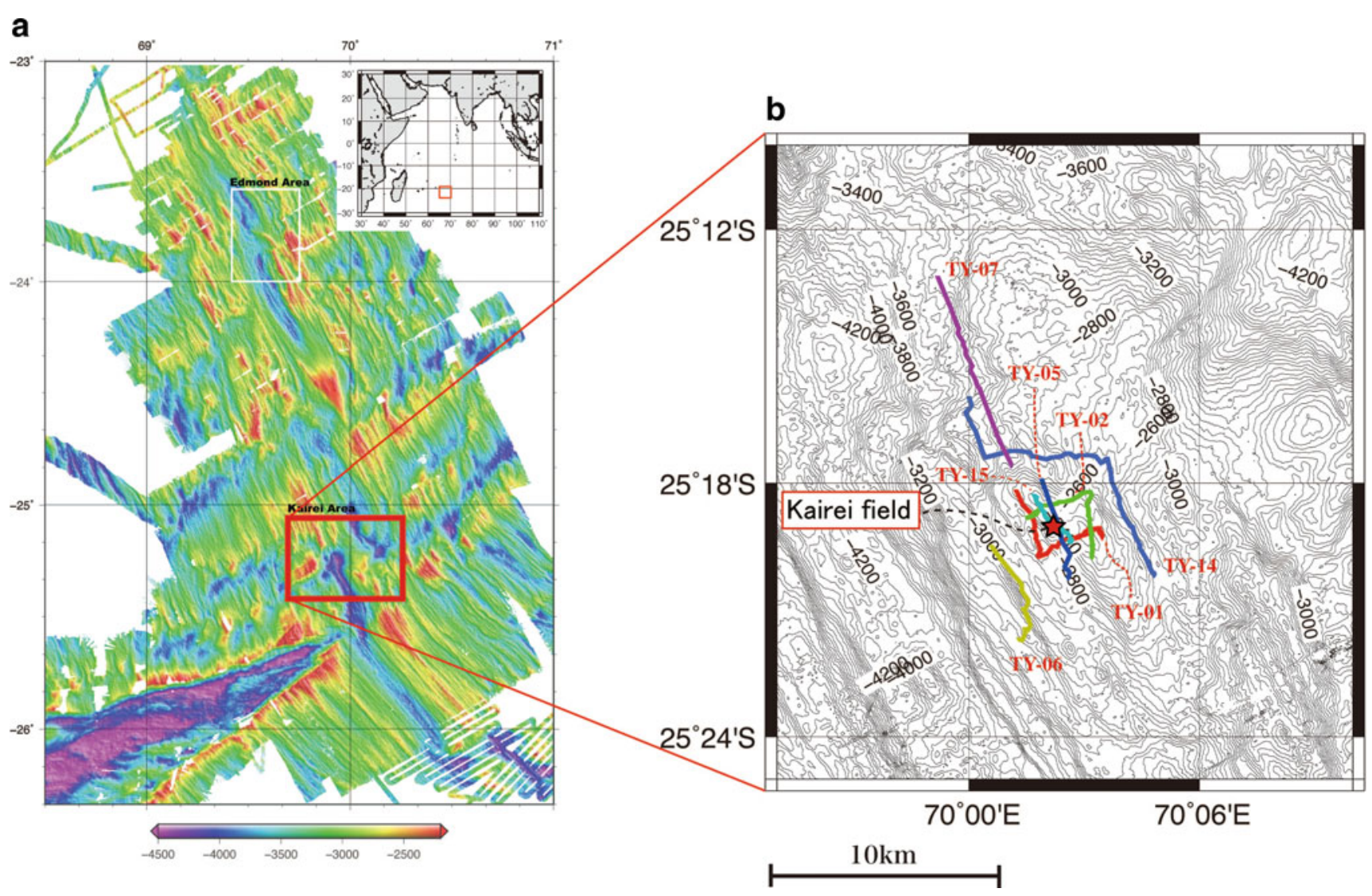

Fig. 15.1 (a) Bathymetric map and locations of hydrothermal fields of Central Indian Ridge. (b) Tow-yo survey lines around Kairei field during KH10-6 cruise in 2010

(Noguchi et al. 2011). The CTDT-CMS system was controlled onboard ship with a CTD deck unit (SBE 11plus, SeaBird Electronics Inc.). A total of 22 Niskin-X bottles (12-L sample volume, General Oceanics) were used at each hydrocast for water sampling. A transponder (Kaiyo Densi Co., Ltd.) was used with a Super Short BaseLine (SSBL) sub-navigation system (Model LNS600, Kaiyo Densi Co., Ltd.) to locate the system. All data except for $\mathrm{pH}$ and ORP were monitored onboard in real time. The system was towed at a speed of 0.5 to $1 \mathrm{knot}$, and water samples were collected when the sensors mounted on the CTDT-CMS showed anomalous signals.

\subsubsection{Sample Preparation and Measurements}

Samples for salinity measurements were collected in amber glass bottles, and salinity was measured with a salinometer (AUTOSAL 8400A, Guildline Instruments). IAPSO standard seawater (Batch P145, Ocean Scientific International Ltd.) was used as the reference sample for standardization (Geiskes et al. 1991). Dissolved oxygen was determined by the Winkler method (Strickland and Parsons 1968), and pH, alkalinity, and dissolved inorganic carbon (DIC) were measured by continuous $0.1 \mathrm{M} \mathrm{HCl}$ titration with $\mathrm{pH}$ monitoring using an automatic titrator (TLED-09, Kimoto Electric, Co., Ltd.). Alkalinity, DIC, and $\mathrm{pH}$ values were determined from the titration data by non-linear leastsquares calculations (Dickson et al. 2007). Samples for $\mathrm{H}_{2}$ and $\mathrm{CH}_{4}$ were collected in $125 \mathrm{~mL}$ volume glass vials without any air bubbles and sterilized by adding $0.5 \mathrm{~mL}$ of saturated $\mathrm{HgCl}_{2}$ solution. For the samples for $\mathrm{H}_{2}$, we used an amber bottle to reduce photochemical oxygenation. Molecular hydrogen was analyzed by a gas chromatograph equipped with a trace reduced gas detector (TRD-1, Round Science Inc.) within $6 \mathrm{~h}$ after sample retrieval on board according to an analytical method described in Kawagucci et al. (2010) with a slight modification. The dissolved gas concentration was calculated from the measured gas mixing ratio in the headspace using the solubility coefficient for $\mathrm{H}_{2}$ (Wiesenburg and Guinasso 1979). The overall analytical uncertainties and detection limits were estimated to be $\pm 10 \%$ and $0.5 \mathrm{nM}$ by repeated analyses of an actual seawater sample and a barometrically diluted commercial standard gas (GL Science). Samples for dissolved $\mathrm{CH}_{4}$ was stored at $4{ }^{\circ} \mathrm{C}$ and brought back to the laboratory. $\mathrm{CH}_{4}$ in the water samples were analyzed by a gas chromatograph (GC-8A, Shimadzu) equipped with a flame ionization 
detector, using a method similar to that described by Tsurushima et al. (1996). The standard deviation, based on repeated analyses of the seawater sample, was $<3 \%$ at a concentration of $3 \mathrm{nM}$.

Microbial cell densities were determined by a flow cytometer (Cell Lab Quanta MPL, Beckman Coulter Inc.) equipped with $488 \mathrm{~nm}$ laser. Seawater samples were aseptically transferred to disposable plastic tubes and stored at $4{ }^{\circ} \mathrm{C}$ until analysis. Microbial cells in the samples were stained with SYBR Green II at a final concentration of $4 \times$ (Molecular Probes Inc.) for $20 \mathrm{~min}$ (Marie et al. 1997) and a $50 \mu \mathrm{L}$ of the sample was injected into the flow cytometer on board.

Microbial phylotype composition in the seawater was determined by the $16 \mathrm{~S}$ rRNA gene sequencing (Sunamura et al. 2004; Nunoura et al. 2012). Hydrothermal plume samples S05ty-11, S15ty-12, and S14ty-6 and a background/reference sample S06ty-5 were used for deepsequencing analysis. Sample S05ty-11 was collected closest to the Kairei hydrothermal field, and sample S14ty-11 was collected farthest from the vents. Microbial cells in a $5 \mathrm{~L}$ seawater sample were collected on a $0.2-\mu \mathrm{m}$-pore-size membrane filter (type GV, Millipore) through filtration. The filter samples were preserved in a deep freezer at $-80{ }^{\circ} \mathrm{C}$ and brought back to the laboratory. DNA in the cells on the filter samples was extracted by using commercial DNA extraction kits (UltraClean Soil DNA Isolation Kit, MO-Bio Laboratories Inc.) according to the manufacturer's instructions. The $16 \mathrm{~S}$ rRNA genes were amplified by polymerase chain reaction (PCR) using LA Taq (TaKaRa Bio Inc.) with a program of $95^{\circ} \mathrm{C}$ for $5 \mathrm{~min}$, followed by 35 cycles of $95{ }^{\circ} \mathrm{C}$ for $30 \mathrm{~s}, 52^{\circ} \mathrm{C}$ for $20 \mathrm{~s}$, and $72{ }^{\circ} \mathrm{C}$ for $30 \mathrm{~s}$, and a final extension at $72{ }^{\circ} \mathrm{C}$ for $10 \mathrm{~min}$. We used the primer set Univ530F and Univ907R, modified from Nunoura et al. (2012). An adaptor sequence for the FLX sequencer and a DNA tag for discrimination of each sample were attached to the $5^{\prime}$ end of the forward primer. Another adaptor sequence for the FLX sequencer was attached to the $5^{\prime}$ end of the reverse primer. After the PCR amplicons were electrophoresed on agarose gel, the target bands were extracted and purified with a QIAquick Gel Purification Kit (Qiagen) according to the manufacturer's instructions. The mixture of purified $16 \mathrm{~S}$ rRNA gene amplicons were sequenced with an FLX sequencer at Takara Dragon Genomics Center. The sequences were separated by the DNA tags and trimmed and chimeras were removed, and then analyzed using mothur software with the Schloss standard operation procedure (SOP) (Schloss et al. 2009, 2011).

\subsection{Results and Discussion}

\subsubsection{Hydrothermal Plume Around the Kairei Field}

Table 15.1 shows the chemical properties and microbial cell densities of seawater samples collected by CTD-CMS sampler. In Fig. 15.2, we show the two-dimensional distributions of turbidity, temperature, and dissolved $\mathrm{Mn}$ anomalies observed by tow-yo survey line S15ty around the Kairei field. The results from the other tow-yo survey lines are also shown in Suppl. 15.2-15.7 (supplementary data). The hydrothermal plume was determined based on the higher turbidity $(\triangle \mathrm{FTU})$ in the plume than in the ambient nonplume seawater (Suppl. 15.8).

The hydrothermal plume around Kairei field arose from the seafloor up to approximately 2,150-m water depth below the sea surface and the thickness of the non-buoyant plume was approximately $120 \mathrm{~m}$ (Fig. 15.2). Gamo et al. (1996, 2001) and Zhu et al. (2008) detected the hydrothermal plume based on the turbidity anomalies around Kairei field in September 1993 (Gamo et al. 1996), in August 2000 (Gamo et al. 2001), and in December 2005 (Zhu et al. 2008), respectively. The previous studies also reported the upper limit depth of hydrothermal plume at $2,150 \mathrm{~m}$ below sea surface (Gamo et al. 1996, 2001; Zhu et al. 2008). The variation of seawater density is caused by the temperature and salinity. Considering that the maximum height of hydrothermal plume is regulated by the density of the end-member hydrothermal fluid and vertical distribution of the density in the ambient seawater, the hydrothermal fluid chemistry and discharging pattern of the Kairei field would have been stable for at least 17 years.

The horizontal distribution of the hydrothermal plume based on the turbidity anomalies showed the Kairei hydrothermal plume was spread to north or northwest direction from the vents in Kairei field (Suppl. 15.2-15.7). Figure 15.3 demonstrated the horizontal distribution of $\mathrm{CH}_{4}, \mathrm{H}_{2}$, and microbial cell density in the hydrothermal plume (from 2,144 to $3,341 \mathrm{~m}$ of water depth) using the water sample collected by the CTD-CMS. The maximum concentration of $\mathrm{H}_{2}$ in the hydrothermal plume was $87.6 \mathrm{nM}$ observed near the Kairei field (Table 15.1). The $\mathrm{H}_{2}$ anomaly was observed only close to the hydrothermal vents at Kairei field (Fig. 15.3a). The maximum $\mathrm{CH}_{4}$ concentration of $12.3 \mathrm{nM}$ was observed approximately $14 \mathrm{~km}$ northwest of the Kairei field, which termed "the north plume" (Fig. 15.3b). In this area, we also observed increased microbial cell densities 
Table 15.1 Results of chemical analysis and microbial cell density in seawater samples collected from Kairei field during KH10-6 cruise in 2010

\begin{tabular}{|c|c|c|c|c|c|c|c|c|c|c|c|c|c|}
\hline $\begin{array}{l}\text { CTD } \\
\text { cast }\end{array}$ & $\begin{array}{l}\text { Bottle } \\
\text { no. }\end{array}$ & $\begin{array}{l}\text { Latitude } \\
\left({ }^{\circ} \mathrm{S}\right)\end{array}$ & $\begin{array}{l}\text { Longitude } \\
\left({ }^{\circ} \mathrm{E}\right)\end{array}$ & $\begin{array}{l}\text { Depth } \\
(\mathrm{m})\end{array}$ & $\begin{array}{l}\text { Time } \\
\text { (GMT) }\end{array}$ & $\begin{array}{l}\text { Salinity } \\
\text { (PSU) }\end{array}$ & $\mathrm{pH}_{\mathrm{T}}$ & $\begin{array}{l}\text { Alkalinity } \\
(\mu \mathrm{M})\end{array}$ & $\begin{array}{l}\text { TIC } \\
(\mu \mathrm{M})\end{array}$ & $\begin{array}{l}\mathrm{DO} \\
\left(\mathrm{mg} \mathrm{L}^{-1}\right)\end{array}$ & $\begin{array}{l}\mathrm{H}_{2} \\
(\mathrm{nM})\end{array}$ & $\begin{array}{l}\mathrm{CH}_{4} \\
(\mathrm{nM})\end{array}$ & $\begin{array}{l}\text { Cell count } \\
\left(\text { cell } \mathrm{mL}^{-1}\right)\end{array}$ \\
\hline \multirow{6}{*}{ S01ty } & 01 & 25.31921 & 70.05670 & 2,294 & $15: 47$ & 34.711 & 7.62 & 2,376 & 2,280 & 3.58 & 0.9 & 0.1 & $1.69 \times 10^{4}$ \\
\hline & 02 & 25.31771 & 70.05296 & 2,314 & $16: 25$ & 34.712 & 7.59 & 2,381 & 2,296 & 3.61 & 0.8 & 0.7 & $2.07 \times 10^{4}$ \\
\hline & 03 & 25.31883 & 70.05117 & 2,202 & $16: 39$ & 34.711 & 7.58 & 2,371 & 2,287 & 3.57 & 0.3 & 2.0 & $1.30 \times 10^{4}$ \\
\hline & 04 & 25.32032 & 70.04932 & 2,349 & $17: 53$ & 34.710 & 7.59 & 2,404 & 2,316 & 3.58 & 37.0 & 4.0 & $7.72 \times 10^{3}$ \\
\hline & 05 & 25.32481 & 70.03033 & 2,250 & $18: 21$ & 34.711 & N.A & N.A & N.A & 3.52 & 14.6 & 3.2 & $1.31 \times 10^{4}$ \\
\hline & 06 & 25.31235 & 70.02707 & 2,141 & $19: 20$ & 34.710 & 7.60 & 2,380 & 2,291 & 3.51 & 13.6 & 3.1 & $1.36 \times 10^{4}$ \\
\hline \multirow[t]{6}{*}{ S02ty } & 01 & 25.32504 & 70.05339 & 2,290 & $22: 18$ & 34.708 & 7.607 & 2,380 & 2,291 & 3.55 & 5.8 & 2.4 & $1.37 \times 10^{4}$ \\
\hline & 02 & 25.32004 & 70.05325 & 2,242 & $23: 01$ & 34.706 & 7.614 & 2,380 & 2,288 & 3.53 & 65.5 & 6.2 & $1.33 \times 10^{4}$ \\
\hline & 03 & 25.31596 & 70.05241 & 2,289 & $23: 22$ & 34.710 & 7.612 & 2,377 & 2,288 & 3.52 & 87.6 & 6.2 & $1.31 \times 10^{4}$ \\
\hline & 04 & 25.30835 & 70.03345 & 2,350 & $1: 44$ & 34.711 & 7.594 & 2,376 & 2,287 & 3.52 & 3.2 & 1.1 & $9.42 \times 10^{3}$ \\
\hline & 05 & 25.31050 & 70.03056 & 2,316 & 2:01 & 34.712 & 7.599 & 2,375 & 2,286 & 3.45 & 4.4 & 3.9 & $1.32 \times 10^{4}$ \\
\hline & 06 & 25.31348 & 70.02512 & 2,250 & $2: 26$ & 34.717 & 7.603 & 2,376 & 2,289 & 3.51 & 4.9 & 3.7 & $8.10 \times 10^{3}$ \\
\hline \multirow[t]{13}{*}{ S05ty } & 01 & 25.33348 & 70.04157 & 2,750 & $14: 29$ & 34.712 & 7.668 & 2,374 & 2,276 & 3.51 & 0.3 & 0.6 & $1.01 \times 10^{4}$ \\
\hline & 02 & 25.33343 & 70.04160 & 2,750 & $14: 29$ & 34.712 & 7.669 & 2,374 & 2,276 & 3.56 & 0.7 & 0.6 & $9.48 \times 10^{3}$ \\
\hline & 03 & 25.32855 & 70.04385 & 2,620 & $14: 59$ & 34.711 & 7.662 & 2,374 & 2,277 & 3.57 & 0.6 & 0.7 & $9.32 \times 10^{3}$ \\
\hline & 04 & 25.32740 & 70.04330 & 2,250 & $15: 06$ & 34.710 & 7.657 & 2,364 & 2,276 & 3.49 & 10.1 & 1.8 & $1.06 \times 10^{4}$ \\
\hline & 05 & 25.32318 & 70.04033 & 2,360 & $15: 51$ & 34.712 & 7.645 & 2,369 & 2,300 & 3.48 & 19.4 & 7.2 & $1.06 \times 10^{4}$ \\
\hline & 06 & 25.32047 & 70.03976 & 2,320 & $16: 10$ & 34.710 & 7.654 & 2,393 & 2,275 & 3.49 & 5.8 & 3.4 & $9.32 \times 10^{3}$ \\
\hline & 07 & 25.32014 & 70.03966 & 2,260 & $16: 13$ & 34.709 & 7.65 & 2,369 & 2,269 & 3.55 & 33.8 & 2.7 & $6.34 \times 10^{3}$ \\
\hline & 08 & 25.31882 & 70.03852 & 2,320 & $16: 29$ & 34.710 & 7.651 & 2,364 & 2,276 & 3.51 & 4.0 & 2.9 & $1.12 \times 10^{4}$ \\
\hline & 09 & 25.31609 & 70.03853 & 2,320 & $16: 45$ & 34.704 & 7.657 & 2,368 & 2,269 & 3.48 & 1.2 & 2.0 & $1.41 \times 10^{4}$ \\
\hline & 10 & 25.31402 & 70.03773 & 2,310 & $17: 01$ & 34.712 & 7.664 & 2,364 & 2,274 & 3.51 & 46.1 & 3.7 & $1.13 \times 10^{4}$ \\
\hline & 11 & 25.30945 & 70.03563 & 2,320 & $17: 37$ & 34.712 & 7.642 & 2,971 & 2,276 & 3.48 & 38.2 & 4.5 & $1.77 \times 10^{4}$ \\
\hline & 12 & 25.30918 & 70.03551 & 2,260 & $17: 39$ & 34.710 & 7.657 & 2,368 & 2,278 & 3.48 & 0.2 & 4.3 & $1.76 \times 10^{4}$ \\
\hline & 13 & 25.29885 & 70.03158 & 2,260 & $18: 44$ & 34.734 & 7.67 & 2,371 & 2,271 & 3.50 & 1.0 & 3.3 & $2.48 \times 10^{4}$ \\
\hline \multirow[t]{4}{*}{ S06ty } & 01 & 25.36184 & 70.02345 & 3,341 & $21: 59$ & 34.714 & 7.671 & 2,371 & 2,272 & 3.60 & $\mathrm{BD}$ & 0.5 & $1.38 \times 10^{4}$ \\
\hline & 02 & 25.35187 & 70.02498 & 2,260 & 23:03 & 34.711 & 7.663 & 2,364 & 2,269 & 3.49 & $\mathrm{BD}$ & 1.2 & $1.40 \times 10^{4}$ \\
\hline & 03 & 25.34604 & 70.02547 & 2,800 & $23: 38$ & 34.713 & 7.675 & 2,376 & 2,273 & 3.80 & 2.9 & 0.8 & $9.06 \times 10^{3}$ \\
\hline & 04 & 25.34004 & 70.02269 & 2,850 & $0: 19$ & 34.714 & 7.683 & 2,368 & 2,268 & & $\mathrm{BD}$ & 0.7 & $9.14 \times 10^{3}$ \\
\hline \multirow[t]{9}{*}{ S07ty } & 01 & 25.30796 & 70.06516 & 2,300 & $18: 28$ & 34.709 & 7.68 & 2,367 & 2,270 & 3.50 & 0.1 & 1.8 & $2.07 \times 10^{4}$ \\
\hline & 02 & 25.28945 & 70.04014 & 2,500 & $21: 16$ & 34.711 & 7.687 & 2,373 & 2,272 & 3.53 & $\mathrm{BD}$ & 1.1 & $8.44 \times 10^{3}$ \\
\hline & 03 & 25.29034 & 70.03228 & 2,460 & $21: 44$ & 34.712 & 7.682 & 2,373 & 2,272 & 3.52 & $\mathrm{BD}$ & 1.2 & $1.45 \times 10^{4}$ \\
\hline & 04 & 25.28732 & 70.02214 & 2,300 & $22: 22$ & 34.710 & 7.682 & 2,368 & 2,269 & 3.50 & 0.7 & 2.8 & $1.87 \times 10^{4}$ \\
\hline & 05 & 25.28947 & 70.00650 & 2,280 & 23:01 & 34.708 & 7.677 & 2,366 & 2,267 & 3.51 & $\mathrm{BD}$ & 2.5 & $2.0 \times 10^{4}$ \\
\hline & 06 & 25.28871 & 70.00615 & 2,200 & 23:04 & 34.708 & 7.574 & 2,365 & 2,281 & 3.48 & 0.1 & 6.5 & $1.41 \times 10^{4}$ \\
\hline & 07 & 25.27991 & 70.00283 & 2,200 & $23: 43$ & 34.710 & 7.652 & 2,384 & 2,290 & 3.50 & $\mathrm{BD}$ & 2.5 & $1.65 \times 10^{4}$ \\
\hline & 08 & 25.27262 & 69.99861 & 2,168 & $0: 24$ & 34.708 & 7.688 & 2,360 & 2,263 & 3.50 & 0.4 & 3.4 & $1.74 \times 10^{4}$ \\
\hline & 09 & 25.26683 & 70.00009 & 2,200 & $1: 01$ & 34.710 & 7.667 & 2,366 & 2,268 & 3.56 & 0.9 & 3.3 & $1.95 \times 10^{4}$ \\
\hline \multirow[t]{9}{*}{ S14ty } & 01 & 25.26408 & 70.00490 & 2,850 & $17: 25$ & 34.904 & 7.648 & 2,385 & 2,292 & 3.61 & 0.7 & 0.94 & $1.72 \times 10^{4}$ \\
\hline & 02 & 25.25796 & 70.00176 & 2,200 & $18: 24$ & 34.709 & 7.663 & 2,386 & 2291 & 3.62 & $\mathrm{BD}$ & 1.33 & $1.29 \times 10^{4}$ \\
\hline & 03 & 25.25481 & 70.00121 & 2,600 & $18: 50$ & 34.710 & 7.661 & 2,386 & 2,294 & 3.56 & 2.5 & 1.50 & $1.24 \times 10^{4}$ \\
\hline & 04 & 25.25434 & 70.00141 & 2,500 & $18: 53$ & 34.711 & 7.673 & 2,376 & 2,283 & 3.56 & 0.5 & 9.25 & $2.11 \times 10^{4}$ \\
\hline & 05 & 25.24991 & 69.99868 & 2,450 & $19: 31$ & 34.710 & 7.665 & 2,384 & 2,289 & 3.76 & 0.3 & 5.48 & $1.64 \times 10^{4}$ \\
\hline & 06 & 25.24444 & 69.99727 & 2,550 & $20: 06$ & 34.711 & 7.662 & 2,385 & 2,295 & 3.55 & 0.5 & 12.27 & $2.20 \times 10^{4}$ \\
\hline & 07 & 25.23774 & 69.99451 & 2,600 & $20: 44$ & 35.141 & 7.667 & 2,451 & 2,356 & 4.47 & 0.5 & 4.79 & $4.80 \times 10^{4}$ \\
\hline & 08 & 25.23049 & 69.99164 & 2,700 & $21: 20$ & 34.710 & 7.916 & 2,323 & 2,127 & 3.59 & $\mathrm{BD}$ & 1.57 & $1.21 \times 10^{4}$ \\
\hline & 09 & 25.21891 & 69.98633 & 2,000 & $22: 11$ & 34.694 & 7.663 & 2,366 & 2,275 & 3.42 & $\mathrm{BD}$ & 1.00 & $1.02 \times 10^{4}$ \\
\hline \multirow[t]{6}{*}{ S15ty } & 01 & 25.32301 & 70.04336 & 2,400 & $14: 00$ & 34.709 & 7.587 & 2,377 & 2,274 & 3.50 & 1.7 & 1.50 & $2.10 \times 10^{4}$ \\
\hline & 02 & 25.32255 & 70.04448 & 2,400 & $14: 19$ & 34.705 & 7.587 & 2,375 & 2,274 & 3.54 & 0.2 & 1.37 & $1.64 \times 10^{4}$ \\
\hline & 03 & 25.32205 & 70.04413 & 2,300 & $14: 21$ & 34.706 & 7.574 & 2,369 & 2,272 & 3.48 & 0.6 & 1.31 & $1.49 \times 10^{4}$ \\
\hline & 04 & 25.32062 & 70.04308 & 2,416 & $14: 36$ & 34.709 & N.A & 2,401 & 2,276 & 3.48 & 1.7 & 1.65 & $7.94 \times 10^{3}$ \\
\hline & 05 & 25.31912 & 70.04133 & 2,250 & $14: 56$ & 34.707 & 7.665 & 2,421 & 2280 & 3.47 & 7.8 & 2.78 & $1.55 \times 10^{4}$ \\
\hline & 06 & 25.31732 & 70.03962 & 2,270 & $15: 15$ & 34.708 & 7.660 & 2,388 & 2,264 & 3.46 & 12.2 & 2.54 & $1.54 \times 10^{4}$ \\
\hline
\end{tabular}


Table 15.1 (continued)

\begin{tabular}{|c|c|c|c|c|c|c|c|c|c|c|c|c|c|}
\hline $\begin{array}{l}\text { CTD } \\
\text { cast }\end{array}$ & $\begin{array}{l}\text { Bottle } \\
\text { no. }\end{array}$ & $\begin{array}{l}\text { Latitude } \\
\left({ }^{\circ} \mathrm{S}\right)\end{array}$ & $\begin{array}{l}\text { Longitude } \\
\left({ }^{\circ} \mathrm{E}\right)\end{array}$ & $\begin{array}{l}\text { Depth } \\
\text { (m) }\end{array}$ & $\begin{array}{l}\text { Time } \\
\text { (GMT) }\end{array}$ & $\begin{array}{l}\text { Salinity } \\
\text { (PSU) }\end{array}$ & $\mathrm{pH}_{\mathrm{T}}$ & $\begin{array}{l}\text { Alkalinity } \\
(\mu \mathrm{M})\end{array}$ & $\begin{array}{l}\text { TIC } \\
(\mu \mathrm{M})\end{array}$ & $\begin{array}{l}\mathrm{DO} \\
\left(\mathrm{mg} \mathrm{L}^{-1}\right)\end{array}$ & $\begin{array}{l}\mathrm{H}_{2} \\
(\mathrm{nM})\end{array}$ & $\begin{array}{l}\mathrm{CH}_{4} \\
(\mathrm{nM})\end{array}$ & $\begin{array}{l}\text { Cell count } \\
\left(\text { cell } \mathrm{mL}^{-1}\right)\end{array}$ \\
\hline & 07 & 25.31595 & 70.03770 & 2,256 & $15: 35$ & 34.708 & 7.648 & 2,389 & 2,270 & 3.46 & 22.0 & 3.17 & $1.00 \times 10^{4}$ \\
\hline & 08 & 25.31587 & 70.03749 & 2,225 & $15: 36$ & 34.707 & 7.676 & 2,419 & 2,292 & 3.46 & 33.5 & 3.60 & $6.22 \times 10^{3}$ \\
\hline & 09 & 25.31501 & 70.03619 & 2,429 & $15: 49$ & 34.708 & 7.655 & 2,397 & 2,274 & 3.47 & 5.7 & 1.61 & $9.88 \times 10^{3}$ \\
\hline & 10 & 25.31479 & 70.03594 & 2,240 & $15: 53$ & 34.707 & 7.644 & 2,389 & 2,270 & 3.44 & 21.6 & 3.10 & $1.07 \times 10^{4}$ \\
\hline & 11 & 25.31483 & 70.03507 & 2,426 & $16: 07$ & 34.709 & 7.640 & 2,385 & 2,267 & 3.46 & 4.4 & 1.44 & $7.96 \times 10^{3}$ \\
\hline & 12 & 25.31207 & 70.03290 & 2,400 & $16: 30$ & 34.708 & 7.633 & 2,388 & 2,268 & 3.49 & 8.9 & 1.77 & $9.72 \times 10^{3}$ \\
\hline & 13 & 25.31065 & 70.03162 & 2,400 & $16: 48$ & 34.707 & N.A & N.A & N.A & 3.45 & 21.5 & 2.25 & $1.40 \times 10^{4}$ \\
\hline & 14 & 25.30832 & 70.03059 & 2,117 & $17: 11$ & 34.706 & 7.641 & 2,390 & 2,273 & 3.42 & 4.2 & 1.63 & $1.37 \times 10^{4}$ \\
\hline & 15 & 25.30768 & 70.02939 & 2,364 & $17: 25$ & 34.708 & 7.642 & 2,383 & 2266 & 3.48 & 10.0 & & $8.68 \times 10^{3}$ \\
\hline & 16 & 25.30585 & 70.02952 & 2,400 & $17: 41$ & 34.710 & 7.661 & 2,398 & 2,272 & 3.46 & 1.4 & 1.18 & $8.90 \times 10^{3}$ \\
\hline & 17 & 25.30573 & 70.02936 & 2,370 & $17: 43$ & 34.708 & 7.623 & N.A & 2,281 & 3.47 & 3.2 & 1.63 & $7.32 \times 10^{3}$ \\
\hline & 18 & 25.30560 & 70.02919 & 2,340 & $17: 44$ & 34.707 & 7.658 & N.A & N.A & 3.43 & 10.2 & 2.31 & $9.22 \times 10^{3}$ \\
\hline & 19 & 25.30548 & 70.02903 & 2,310 & $17: 45$ & 34.709 & 7.618 & 2,376 & 2,264 & 3.43 & 2.1 & 1.66 & $1.19 \times 10^{4}$ \\
\hline & 20 & 25.30536 & 70.02887 & 2,280 & $17: 47$ & 34.708 & N.A & N.A & N.A & 3.49 & 4.5 & 1.78 & $1.17 \times 10^{4}$ \\
\hline & 21 & 25.30524 & 70.02871 & 2,250 & $17: 47$ & 34.707 & N.A & N.A & N.A & 3.49 & 3.4 & 1.55 & $9.50 \times 10^{3}$ \\
\hline & 22 & 25.30511 & 70.02854 & 2,220 & $17: 49$ & 34.707 & 7.609 & 2,371 & 2,261 & 3.45 & 0.4 & 1.29 & $8.22 \times 10^{3}$ \\
\hline
\end{tabular}

$N . A$ not analyze, $B D$ below detection limit
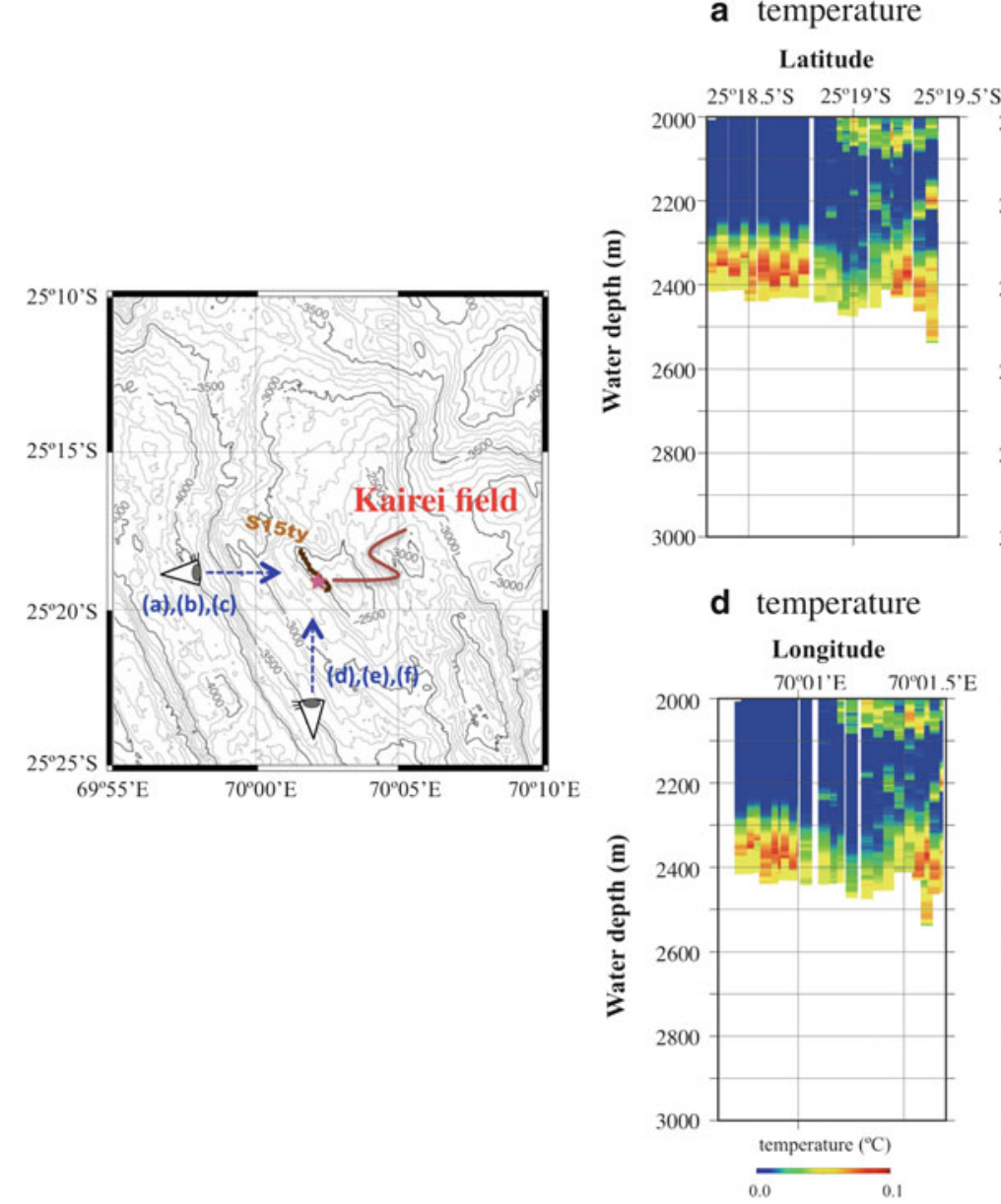

b turbidity

Latitude

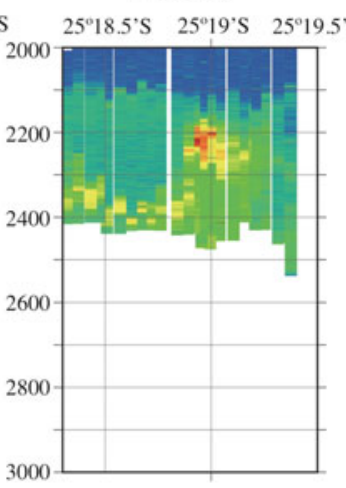

e turbidity

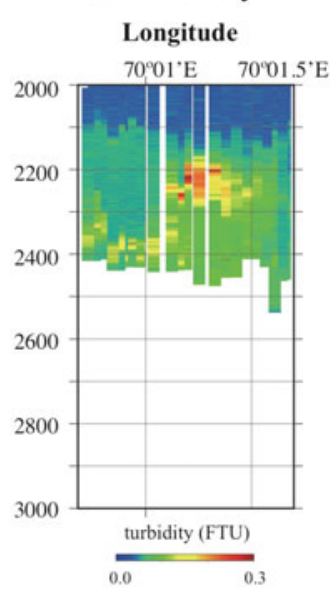

c dissolved $\mathrm{Mn}$

Latitude

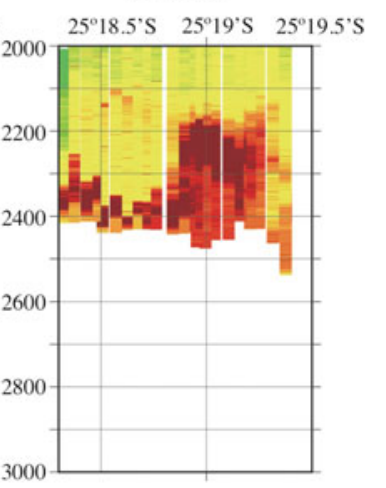

f dissolved Mn

Longitude

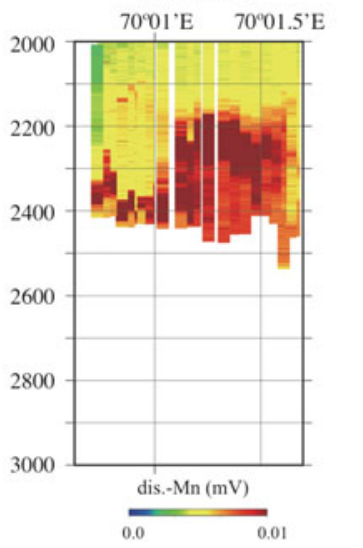

Fig. 15.2 Two-dimensional distribution of temperature (a, d), turbidity (b, e), and dissolved manganese (c, e) anomalies during Tow-yo survey (S15ty) line 


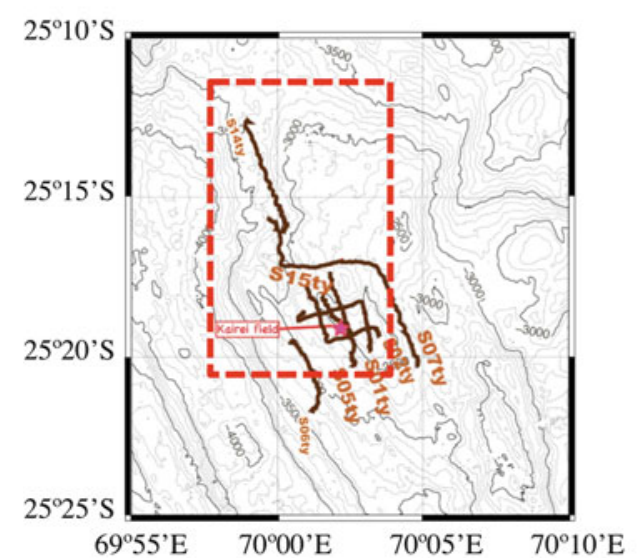

b methane

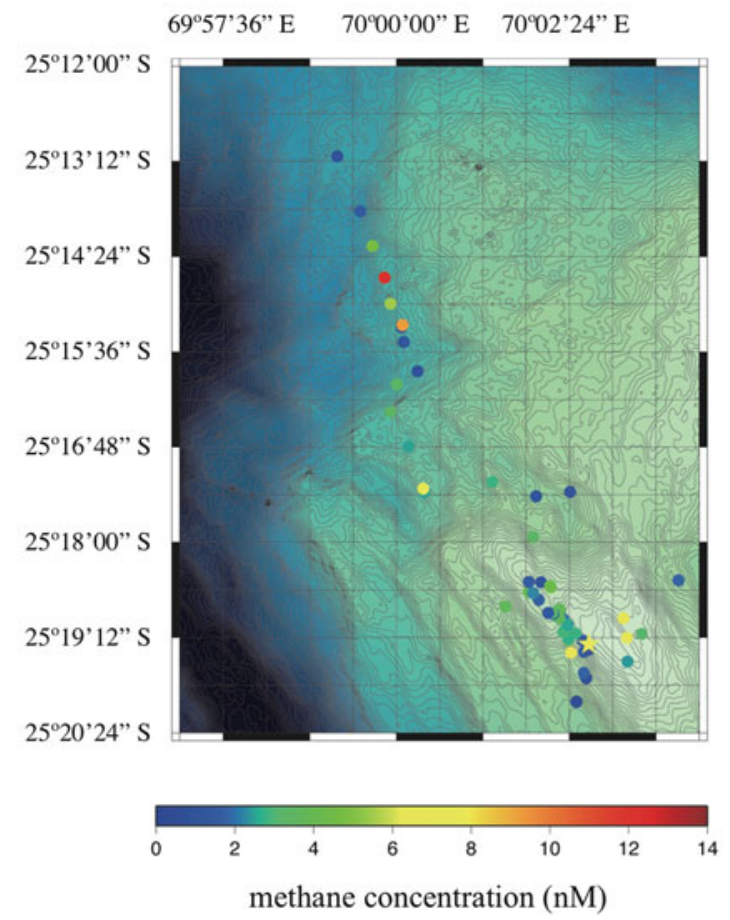

a hydrogen

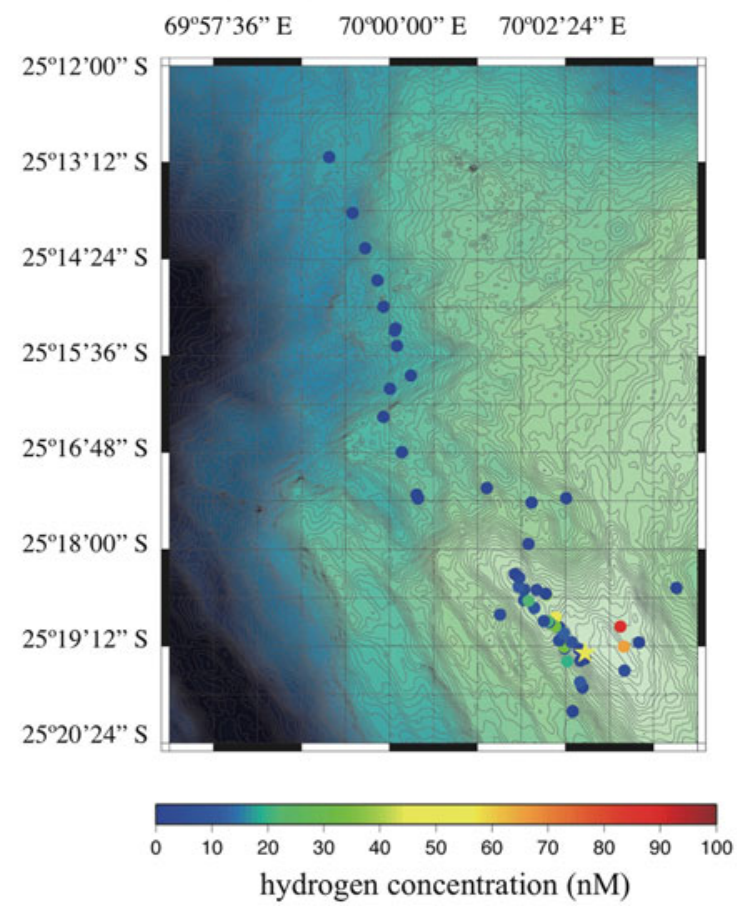

c microbial cell density

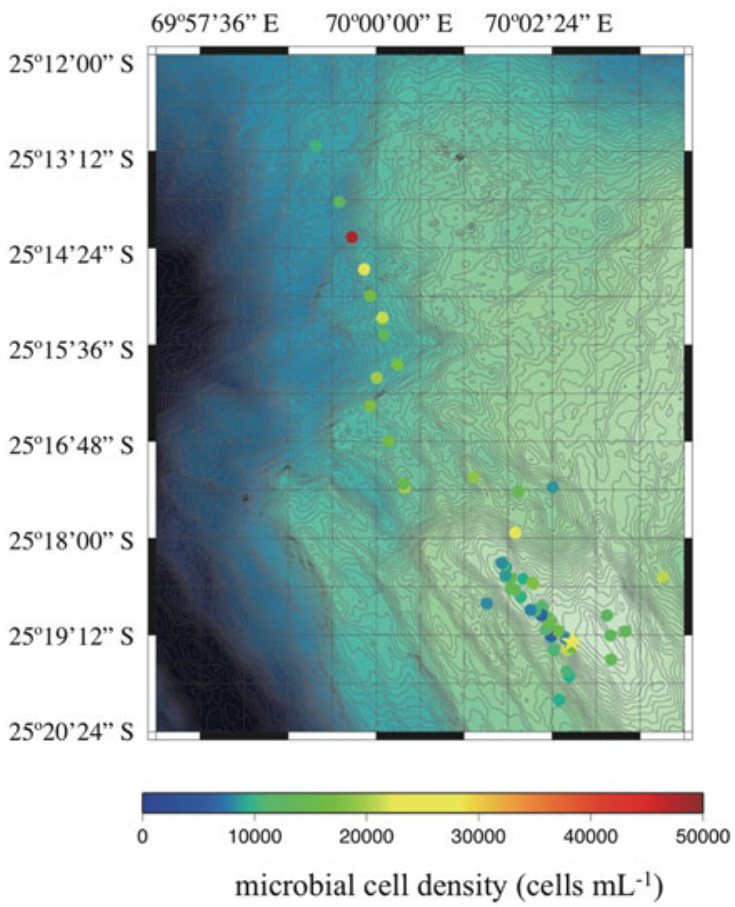

Fig. 15.3 Horizontal distribution of reductive chemical species and microbial cell density around Kairei field, (a) $\mathrm{H}_{2}$, (b) $\mathrm{CH}_{4}$, and (c) microbial cell density

(Fig. 15.3c). In the north plume, the $\mathrm{H}_{2} / \mathrm{CH}_{4}$ ratio in hydrothermal plume sample was completely different from that of the end-member hydrothermal fluid of Kairei field and the plumes near the Kairei field (Fig. 15.4). High and rapid $\mathrm{H}_{2}$ consumption rate by microorganisms in water column (half time of $<1$ day) (e.g., Scranton et al. 1984) may cause the restricted distribution of the $\mathrm{H}_{2}$ anomaly very close to the Kairei field. The $\mathrm{CH}_{4}$ concentration of the north plume was 


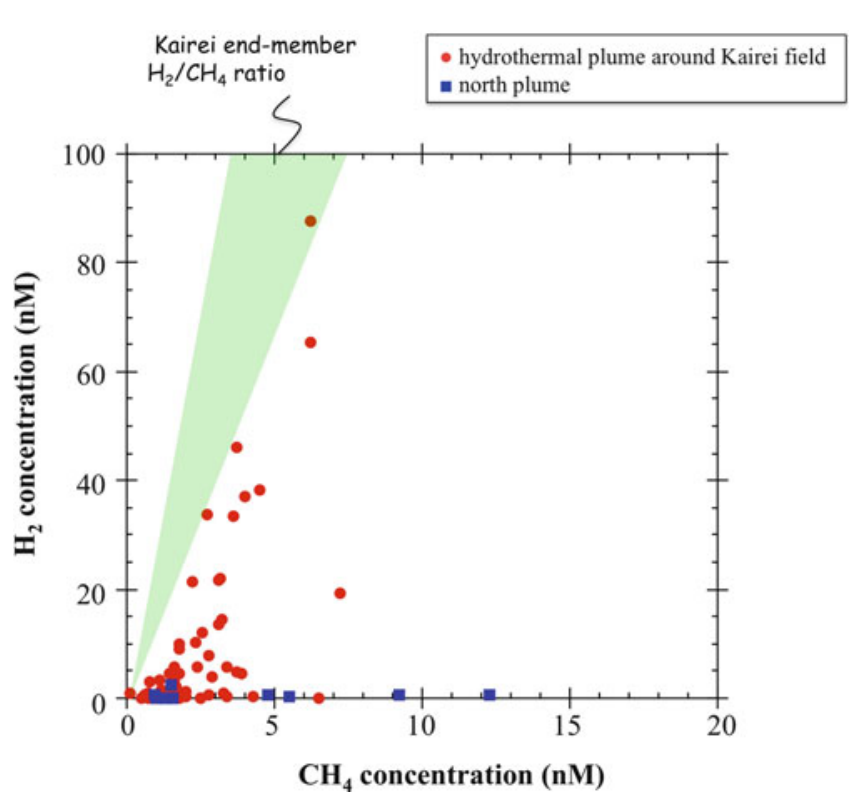

Fig. 15.4 $\mathrm{CH}_{4}$ concentration vs. $\mathrm{H}_{2}$ concentration in sub-sampled water taken by Tow-yo survey. $\mathrm{H}_{2} / \mathrm{CH}_{4}$ ratios of end member compositions of hydrothermal fluids sampled from Kairei Field (15 to 30 ) were estimated by using the data reported by Kumagai et al. (2008)

higher than those of the plume around Kairei field. One of the possible explanations for the high $\mathrm{CH}_{4}$ concentration in the north plume would be caused by different hydrothermal end-member fluid. Gamo et al. (2001) predicted the existence of another hydrothermal vent, which has different trend of $\mathrm{CH}_{4}$ and turbidity ratio, at the north area of Kairei field. The location of north plume, we observed in this time, is farther than that of the plume reported by Gamo et al. (2001). Another possibility of the high $\mathrm{CH}_{4}$ concentration is in situ $\mathrm{CH}_{4}$ production under either anaerobic or aerobic conditions. The anaerobic $\mathrm{CH}_{4}$ production using $\mathrm{H}_{2}$ and $\mathrm{CO}_{2}$ may affect the low $\mathrm{H}_{2}$ and high $\mathrm{CH}_{4}$ concentration in the north plume, however, this microbial reaction is generally restricted in the strict anaerobic condition. The dissolved oxygen concentration in the plume is more than $3 \mathrm{mg} \mathrm{L}^{-1}$, which indicates that anaerobic methane production is hard to occur. Moreover, our deep-sequencing analysis did not detect any phylotypes related to methanogenic archaea in more than 10,000 amplicons of 16S rRNA gene sequences. Karl et al. (2008) reported that the aerobic $\mathrm{CH}_{4}$ production in water column via the decomposition of methylphosphonate under the phosphate-depleted condition during microbial growth. The general abyssal seawater is rich in phosphate, however, phosphate in the plume is removed from seawater due to the absorption to the particles of hydrothermal plume (German and Von Damm 2003). We need further exploration for the methylphosphonate
Table 15.2 16S rRNA gene composition of representative microbial community in hydrothermal plumes by deep-sequencing

\begin{tabular}{lcccl}
\hline & $\begin{array}{l}\text { S14ty-6 } \\
(\%)\end{array}$ & $\begin{array}{l}\text { S15ty-12 } \\
(\%)\end{array}$ & $\begin{array}{l}\text { S05ty-1 } \\
(\%)\end{array}$ & $\begin{array}{l}\text { S06ty-5 } \\
(\%)\end{array}$ \\
\hline Specific microbes & & & & \\
\hline SUP05 & 2.8 & 3.2 & 16.1 & 8.8 \\
\hline Methylotroph & 0.1 & 0.0 & 3.1 & 0.0 \\
\hline $\begin{array}{l}\text { Methlylococcus } \\
\text { (unclassified) }\end{array}$ & 0.0 & 0.0 & 2.8 & 0.0 \\
Methylobacter & 0.1 & 0.0 & 0.3 & 0.0 \\
\hline Methylophilaceae & 0.0 & 0.0 & 0.0 & 0.0 \\
\hline Alcanivorax & 15.0 & 17.6 & 0.8 & 0.6 \\
\hline Epsiron & 4.9 & 0.8 & 10.6 & 1.7
\end{tabular}

concentration and aerobic $\mathrm{CH}_{4}$ production rate in the hydrothermal plume.

\subsubsection{Microbial Populations and Communities}

Around the Kairei field, the microbial cell density in seawater samples varied from $0.6 \times 10^{4}$ to $2.5 \times 10^{4}$ cells $\mathrm{ml}^{-1}$ (Table 15.1, Fig. 15.3c). At a reference site, where is approximately $80 \mathrm{~km}$ north of Kairei field, microbial cell density varied from $0.9 \times 10^{4}$ to $1.4 \times 10^{4}$ cells ml $^{-1}$ (not shown in Table 15.1). The microbial cell density in S05ty-11, S15ty-12, and S14ty-6 was the same as, lower than, and higher than the density in the background/reference S06ty-5 sample, respectively. Microbial cell abundance in the hydrothermal plume was up to three fold higher than that in the abyssal seawater. The increase of microbial cell density was comparable to previous studies (Sunamura et al. 2004; Lam et al. 2004). Samples from near the hydrothermal vents had lower microbial cell densities compared to the reference site, whereas the densities in non-buoyant plume samples were larger than the reference site. The non-buoyant plume was defined based on the horizontal distribution of the turbidity anomaly. We compared the microbial cell densities with physico-chemical properties (e.g. $\mathrm{pH}, \mathrm{H}_{2}, \mathrm{CH}_{4}$, temperature, alkalinity, and dissolved $\mathrm{Mn}$ ) but we found no strong correlations.

The microbial 16S rRNA gene phylotype compositions showed that the sequences of Phylum Proteobacteria accounted for about half of the total sequences in the reference sample (S06ty-5), whereas the proportion was increased in the plume samples (Table 15.2 and Suppl. 15.9). Phylogenetic analysis of 16S rRNA gene sequences suggested that potential chemolitho(auto)trophs such as SUP05 (Thioglobus), aerobic methanotrophs (Methylococcus and Methylobacter), Epsilonproteobacteria, and Alcanivorax thrived in the plume (Table 15.2). The SUP05 phylotypes, which are known as sulfur oxidizers (Marshall and Morris 2012) and/or possibly hydrogen oxidizers (Petersen et al. 2011; Anantharamana et al. 
2013), accounted for $16.1 \%$ of the total $16 \mathrm{~S}$ rRNA gene sequences in S05ty-11, the sample collected closest to the Kairei vents. The proportion of SUP05 in the total microbial community decreased with distance from the vents, from $16.1 \%$ in S05ty-11 near the vents to $2.8 \%$ in S14ty-6, respectively. The proportion of the epsilonproteobacterial phylotypes, possible sulfur and/or hydrogen oxidizers, was $10.6 \%, 0.8 \%$, and $4.9 \%$ in S05ty-11, S15ty-12, and S14ty6, respectively. Alcanivorax members, which are potential hydrocarbon utilizers and sulfur oxidizers, were rare $(0.8 \%)$ in the S05ty-11 plume sample, but it increased to $17.6 \%$ and $15.0 \%$ in Sty15-12 and Sty14-6, respectively. The phylotypes of aerobic methanotrophs were detected in S05ty11 (3.1\% of the total sequences), but they accounted for less than $0.1 \%$ in the S15ty-12 and S14ty-6 samples. From these results, it is expected that (1) SUP05, Epsilonproteobacteria, and methanotrophs dominate in the early stage of the hydrothermal plume and in the hydrothermal fluid-seawater mixing zone near the vents; (2) at a short distance from the vents, microbial cells are diluted or removed from seawater by adsorption onto hydrothermal plume particles and precipitation; and (3) in the non-buoyant and evolved hydrothermal plume far from the vent, small population increase of heterotrophs and sulfur oxidizers such as Alcanivorax, Alphaproteobacteria, and Alteromonas would occur.

\subsection{Conclusion}

We showed the two different types of hydrothermal plumes during KH10-6 cruise by R/V Hakuho-maru in 2010. One was characterized by high $\mathrm{H}_{2}$ concentration around Kairei field, and the other was identified as low $\mathrm{H}_{2}$, high $\mathrm{CH}_{4}$ and high microbial cell density hydrothermal plume named as the "north plume" found in approximately $14 \mathrm{~km}$ north of the Kairei field. Although the possibility of in situ aerobic methane production could not be denied, the north plume may originate from the yet unidentified hydrothermal fluid source with high $\mathrm{CH}_{4}$ concentration end-member. In the north plume, slightly increases of heterotrophs or potential sulfur oxidizers such as Alcanivorax, Alpha-proteobacteria, and Alteromonas were occured. The little abundance of methanotroph and increased heterotrophs may indicate a chemolithoautotrophicindependent microbial ecosystem in the plume. Removal of chemicals from the hydrothermal plume is one of the important aspects for global chemical flux. Our results showed that microbial cells may be diluted or removed from seawater by adsorption onto hydrothermal plume particles and precipitation in a short distance from the Kairei vents. This indicated that the impacts of the plume microbes on seafloor ecosystem could not be negligible. $\mathrm{A}_{2}$ anomaly was observed only close to the Kairei field and SUP05 and Epsilon-proteobacteria, which are known as potential hydrogen oxidizer, propagate in the early stage of the hydrothermal plume and in the hydrothermal fluid-seawater mixing zone near the vents. These indicated the rapid microbial $\mathrm{H}_{2}$ consumption.

Acknowledgments This work was funded by the Ministry of Education, Culture, Science and Technology (MEXT), Japan, through the Grant-in-Aid for Scientific Research: Project TAIGA (New Scientific Research on Innovative Areas, 20109003) and "Development of new tools for the exploration seafloor resources". We thank the officers and crew of R/V Hakuho-maru and the scientists who participated in the KH10-6 cruise for water sample collection.

Open Access This chapter is distributed under the terms of the Creative Commons Attribution Noncommercial License, which permits any noncommercial use, distribution, and reproduction in any medium, provided the original author(s) and source are credited.

\section{References}

Anantharamana K, Breierb JA, Sheika CS, Dick GJ (2013) Evidence for hydrogen oxidation and metabolic plasticity in widespread deepsea sulfur-oxidizing bacteria. Proc Natl Acad Sci U S A 110:330-335

Baker ET (1990) Hydrothermal plume prospecting: hydrographic and geochemical techniques. In: McMurray GR (ed) Gorda Ridge. Springer, New York, pp 155-167

Dickson AG, Sabine CL, Christian JR (eds) (2007) Guide to best practices for ocean $\mathrm{CO}_{2}$ measurements. North Pacific Marine Science Organization, Sidney, British Columbia, pp. 176. (PIECES Special Publication 3)

Gallant RM, Von Damm KL (2006) Geochemical controls on hydrothermal fluids from the Kairei and Edmond Vent Fields, 23-25ㅇ, Central Indian Ridge. Geochem Geophys Geosys 7(6), Q06018. doi:10.1029/2005GC001067

Gamo T, Nakayama E, Shitashima K, Isshiki K, Obata H, Okamura K, Kanayama S, Oomori T, Koizumi T, Matsumoto S, Hasumoto H (1996) Hydrothermal plumes at the Rodriguez triple junction, Indian ridge. Earth Planet Sci Lett 142:261-270

Gamo T, Chiba H, Yamanaka T, Okudaira T, Hashimoto J, Tsuchida S, Ishibashi J, Kataoka S, Tsunogai U, Okamura K, Sano Y, Shinjo R (2001) Chemical characteristics of newly discovered black smoker fluids and associated hydrothermal plume at the Rodriguez Triple Junction, Central Indian Ridge3. Earth Planet Sci Lett 193:371-379

Geiskes JM, Gamo T, Brumsack H (1991) Chemical methods for interstitial water analysis aboard JOIDES RESOLUTION. ODP Tech. Note 15, Texas A\&M University, $60 \mathrm{pp}$

German CR, Von Damm KL (2003) Hydrothermal processes. In: Henry E (ed) Treatise on geochemistry, vol. 6. Elsevier, $625 \mathrm{pp}$

Hashimoto J, Ohta S, Gamo T, Chiba H, Yamaguchi T, Tsuchida S, Okudaira T, Watabe H, Yamanaka T, Kitazawa M (2001) First hydrothermal vent communities from the Indian Ocean discovered. Zool Sci 18:717-721

Karl DM, Beversdorf L, Björkman KM, Church MJ, Martinez A, Delong EF (2008) Aerobic production of methane in the sea. Nat Geosci 1:473-478

Kawagucci S, Shirai K, Lan TF, Takahata N, Tusunogai U, Sano Y, Gamo T (2010) Gas geochemical characteristics of hydrothermal plumes at the HAKUREI and JADE vent sites, the Izena Cauldron, Okinawa Trough. Geochem J 44:507-518

Kumagai H, Nakamura K, Toki T, Morishita T, Okino K, Ishibashi J, Tsunogai U, Kawagucci S, Gamo T, Shibuya T, Sawaguchi T, Neo N, Joshima M, Sato T, Takai K (2008) Geological background of Kairei 
and Edmond hydrothermal fields along the Central Indian Ridge: Implications of their vent fluid' distinct chemistry. Geofluid 8:239-251

Lam P, Cowen JP, Jones RD (2004) Autotrophic ammonia oxidation in a deep-sea hydrothermal plume. FEMS Microbiol Ecol 47:191-206

Marie D, Partensky F, Jacquet S, Vaulot D (1997) Enumeration and cell cycle analysis of natural populations of marine picoplankton by flow cytome try using the nucleic acid stain SYBR Green I. Appl Environ Microbiol 63:186-193

Marshall KT, Morris RM (2012) Isolation of an aerobic sulfur oxidizer from the SUP05/Arctic96BD-19 clade. ISME J 7(2):452-455

Naganuma T, Otsuki A, Seki H (1989) Abundance and growth-rate of bacterioplankton community in hydrothermal vent plumes of North Fiji Basin. Deep Sea Res A 36:1379-1390

Nakamura K, Morishita T, Bach W, Klein F, Hara K, Okino K, Takai K, Kumagai H (2009) Serpentinized troctolites exposed near the Kairei Hydrothermal Field, Central Indian Ridge: Insights into the origin of the Kairei hydrothermal fluid supporting a unique microbial ecosystem. Earth Planet Sci Lett 280:128-136

Noguchi T, Okamura K, Hatta M, Kimoto H, Suzue T, Ishibashi J, Yamanaka T, Fujii T (2011) Miniaturization of in situ manganese analyzer and its long-term observation at the Wakamiko submarine crater in Kagoshima bay. BUTSURI-TANSA 64(4):291-297 (in Japanese with English abstract)

Nunoura T, Takaki Y, Kazama H, Hirai M, Ashi J, Imachi H, Takai K (2012) Microbial diversity in deep-sea methane seep sediments presented by SSU rRNA gene tag sequencing. Microbs Environ 27(4):382-390

Petersen JM, Zielinski FU, Pape T, Seifert R, Moraru C, Amann R, Hourdez S, Girguis PR, Wankel SD, Barbe V, Pelletier E, Fink D, Borowski C, Bach W, Dubilier N (2011) Hydrogen is an energy source for hydrothermal vent symbioses. Nature 476:176-180

Schloss PD, Westcott SL, Ryabin T, Hall JR, Hartmann M, Hollister EB, Lesniewski RA, Oakley BB, Parks DH, Robinson CJ, Sahl JW, Stres B, Thallinger GG, Van Horn DJ, Weber CF (2009)
Introducing mothur: open-source, platform-independent, community-supported software for describing and comparing microbial communities. Appl Environ Microbiol 75(23):7537-7541

Schloss PD, Gevers D, Westcott SL (2011) Reducing the effects of PCR amplification and sequencing artifacts on 16S rRNA-based studies. PLoS One 6(12):e27310

Scranton MI, Novelli PC, Loud PA (1984) The distribution and cycling hydrogen gas in the waters of two anoxic marine environments. Limnol Ocean 29(5):993-1003

Shitashima K, Kyo M, Koike Y, Henmi H (2002) Development of in situ $\mathrm{pH}$ sensor using ISFET. In: Proceedings of the 2002 international symposium on underwater technology. IEEE/02EX556, pp 106-108

Strickland JDH, Parsons TR (1968) Determination of dissolved oxygen. In: A practical handbook of seawater analysis, vol 167. Fisheries Research Board of Canada, Bulletin, pp 71-75

Sunamura M, Higashi Y, Miyako C, Ishibashi J, Maruyama A (2004) Two bacteria phylotypes are predominant in the Suiyo Seamount hydrothermal plume. Appl Environ Microbiol 70:1190-1198

Tsurushima N, Watanabe S, Tsunogai U (1996) Methane in the East China Sea Water. J Oceanogr 52:221-233

Van Dover CL, Humphris SE, Fornari D, Cavanaugh CM, Collier R, Goffredi SK, Hashimoto J, Lilly MD, Reysenbach AL, Shank TM, Von Damm KL, Banta A, Gallant RM, Götz D, Green D, Hall J, Harmer TL, Hurtado LA, Johnson P, McKiness ZP, Meredith C, Olson E, Pan IL, Turnipseed M, Won Y, Young III CR, Vrijenhoek RC (2001) Biogeography and ecological setting of Indian Ocean hydrothermal vents. Science 294:818-823

Wiesenburg DA, Guinasso NL (1979) Equilibrium solubilities of methane, carbon-monoxide, and hydrogen in water and sea-water. J Chem Eng Data 24:356-360

Winn CD, Karl DM, Massoth GJ (1986) Microorganisms in deep-sea hydrothermal plumes. Nature 320:744-746

Zhu J, Lin J, Guo S, Chen Y (2008) Hydrothermal plume anomalies along the Central Indian Ridge. Chinese Sci Bull 53(16):2527-2535 